\title{
Effects of cyclic tension stress on the apoptosis of osteoclasts in vitro
}

\author{
FENGBO LI, XIAOLEI SUN, BIN ZHAO, JIANXIONG MA, YANG ZHANG, \\ SHUANG LI, YANJUN LI and XINLONG MA \\ Institute of Orthopedics, Tianjin Hospital, Tianjin 300211, P.R. China
}

Received June 27, 2014; Accepted February 4, 2015

DOI: $10.3892 /$ etm.2015.2338

\begin{abstract}
The aim of the present study was to investigate the effect of cyclic tension stress on osteoclast apoptosis in vitro using murine RAW264.7 cells treated with receptor activator of nuclear factor- $\kappa \mathrm{B}$. Using the EF3200 mechanical testing instrument with BioDynamic bioreactor system, cultured osteoclasts which were seeded in a silicone rubber membrane load carrier, were loaded with periodic cyclic stretch microstrain. The induced osteoclasts were subjected to $0,5,10$ and $15 \%$ stretch microstrain for $1 \mathrm{~h}$ daily for three days. The number of tartrate-resistant acid phosphatase-positive osteoclasts and the resorption area were assessed. Osteoclast apoptosis was detected by the Annexin V-fluorescein isothiocyanate (FITC)/propidium iodide binding assay. The mRNA expression of Bcl-2, Bax, caspase-3 and cytochrome $c$ was detected following force loading using reverse transcription-quantitative polymerase chain reaction (RT-qPCR) analysis. Compared with the cells under no cyclic tension stress, the number of osteoclasts and the resorption area were increased in the cells under 10 and $15 \%$ stretch microstrain. The Annexin V binding assay showed that the early apoptosis rate of the 5,10 and $15 \%$ stretch microstrain groups was decreased compared with that of the control group. RT-qPCR results showed that the $\mathrm{Bcl}-2 / \mathrm{Bax}$ ratio was significantly increased in the cells subjected to 5,10 and $15 \%$ stretch microstrain compared with that in the control cells $(\mathrm{P}<0.05)$, while the expression of cytochrome $c$ in the 10 and $15 \%$ stretch microstrain groups was decreased significantly $(\mathrm{P}<0.05)$. No significant
\end{abstract}

Correspondence to: Professor Xinlong Ma or Mr. Xiaolei Sun, Institute of Orthopedics, Tianjin Hospital, 406 Jiefangnan Road, Tianjin 300211, P.R. China

E-mail: 765852368@qq.com

E-mail: davidsun0812@gmail.com

Abbreviations: RANKL, receptor activator of nuclear factor $-\kappa \mathrm{B}$ ligand; TRAP, tartrate-resistant acid phosphatase; NO, nitric oxide; PGE2, prostaglandin E2; M-CSF, macrophage colony-stimulating factor; APAF-1, apoptosis protease activating factor-1

Key words: cyclic tension stress, osteoclast, apoptosis, tartrate-resistant acid phosphatase, mitochondria, RAW264.7 difference was observed between the cytochrome $c$ expression of the 5\% stretch microstrain group and that of the control group ( $\mathrm{P}>0.05)$. The expression of caspase-3 in the 5, 10 and $15 \%$ stretch microstrain groups was decreased significantly compared with that in the control group $(\mathrm{P}<0.05)$. These data suggest that cyclic tension stress can inhibit apoptosis in osteoclasts, possibly by increasing the $\mathrm{Bcl}-2 / \mathrm{Bax}$ ratio, inhibiting the activity of caspase- 3 and downregulating the expression of cytochrome $c$.

\section{Introduction}

Bone modeling and remodeling are maintained by the strictly coupled activities of bone-forming osteoblasts and bone-resorbing osteoclasts $(1,2)$. Mechanical stimulation from weight-bearing activities and muscle contraction plays an important role in bone modeling and remodeling. Weight bearing and muscle contraction stimulate bone formation and remodeling, whereas a lack of these stimuli leads to bone mass loss, and can cause conditions such as disuse osteoporosis (3-5). By contrast, exposure of the bone to supraphysiological loads results in an increase in bone mass in order to withstand the high stress (6-11). The adaptation of bone to mechanical changes occurs due to osteoblasts and osteoclasts, which sense the changes and regulate the function of bone formation and resorption $(12,13)$.

Numerous types of mechanical stimuli, such as hydrostatic or hydrodynamic pressure, fluid shear stress and hypergravity, have been applied to observe the effects on bone-derived cells. Dynamic loading can stimulate human osteoblast proliferation and increase extracellular matrix production (14-16). In addition, mechanical stimulation of osteoblasts has been to shown to increase the release of alkaline phosphatase (ALP) (17), nitric oxide (NO) (18) and prostaglandin E2 (PGE2) (19), and regulate Runt-related transcription factor 2 (Runx2) activation (20).

Osteoclasts are multinucleated cells that branch from the monocyte or macrophage lineage early during the differentiation process (21). The osteoclast formation process includes several steps. Firstly, precursor cells are genetically altered so that the proteins expressed enable cell-cell recognition and attachment. The cells then undergo differentiation to multinucleated pre-osteoclasts, which do not resorb bone. These pre-osteoclasts are finally activated into functional bone 
resorbing osteoclasts by a number of hormones and cytokines, including parathyroid hormone, 1,25-dihydroxyvitamin D3, tumor necrosis factor (TNF) and interleukin-1, which promote osteoclastogenesis. Receptor activator of nuclear factor- $\kappa \mathrm{B}$ (RANKL) and macrophage colony-stimulating factor (M-CSF) are the key factors for osteoclast differentiation $(21,22)$. RANKL is expressed on the membrane surface of osteoblasts and stromal cells and promotes pre-osteoclast fusion into mature osteoclasts. The main role of M-CSF is to induce the pre-osteoclast expression of RANK, which is a receptor of RANKL (21). Mature osteoclasts are tartrate-resistant acid phosphatase (TRAP)-positive cells that cause bone resorption. Studies have reported that mechanical stimulation affects the differentiation and bone resorption function of osteoclasts $(23,24)$.

Mature osteoclasts are terminally differentiated cells and thus do not undergo mitosis. The lifespan of osteoclasts is short, and the cells undergo spontaneous apoptosis. Numerous drugs, such as bisphosphonates, can promote the apoptosis of osteoclasts in order to have an anti-osteoporotic effect $(25,26)$; however, less is known about the effect of mechanical strain on osteoclast apoptosis.

It is known that osteoclasts and osteoblasts are sensitive to mechanical stimulation, which affects the differentiation and bone resorption function of osteoclasts $(23,24)$; however, few studies have reported the effect of cyclic tension stress on osteoclast apoptosis. In the present study, the association between cyclic tension stress and osteoclast apoptosis was investigated using the ElectroForce ${ }^{\circledR} 3200$ mechanical testing instrument (EnduraTEC Systems Group, Bose Corp., Minnetonka, MN, USA) with a BioDynamic ${ }^{\circledR}$ bioreactor system (Bose Corp.).

\section{Materials and methods}

Materials. RAW264.7 cells were purchased from the Institute of Basic Medicine of Peking Union Medical College (Beijing, China). High-glucose Dulbecco's Modified Eagle's Medium (DMEM) and fetal bovine serum (FBS) were obtained from Invitrogen Life Technologies (Carlsbad, CA, USA). RANKL was purchased from PeproTech Co. (Rocky Hill, NJ, USA). The TRAP staining kit was purchased from Sigma-Aldrich (St. Louis, MO, USA), and Silastic Sylgard 184 was obtained from Dow Corning Corp. (Midland, MI, USA). The Annexin V-fluorescein isothiocyanate (FITC) apoptosis detection kit, BD FACSCalibur ${ }^{\mathrm{TM}}$ flow cytometer and flow cytometry and CellQuest sub-analysis software were purchased from BD Biosciences (Franklin Lakes, NJ, USA). The Endura ElectroForce 3200 mechanical testing instrument and BioDynamic bioreactor system were obtained from Bose Corp. For the reverse transcription-quantitative polymerase chain reaction, All-in-One ${ }^{\mathrm{TM}}$ qPCR Mix and All-in-One qPCR Primer (GeneCopoeia, Rockville, MD, USA) were used. Optical and inverted phase contrast microscopes were obtained from Nikon Corp. (Tokyo Japan). $\mathrm{iQ}^{\mathrm{TM}} 5$ Real-Time PCR Detection Elution was purchased from Bio-Rad (Hercules, CA, USA).

Cell culture and osteoclast differentiation. RAW264.7 cells were grown in high-glucose DMEM supplemented with $10 \%$ heat-inactivated FBS and $1 \%$ penicillin-streptomycin at $37^{\circ} \mathrm{C}$ in a humidified atmosphere of $95 \%$ air and $5 \% \mathrm{CO}_{2}$. The differ- entiation of RAW264.7 cells was induced using high-glucose DMEM containing RANKL (100 ng/ml), as described in a previous study (27).

Mechanical loading system. Mechanical loading was performed using a rectangular $(4 \times 2 \times 0.1 \mathrm{~cm})$ silicone rubber membrane developed by our research group. The stress distribution on the surface of the silicone rubber membrane and its biocompatibility were acceptable for cell culture, and the material itself showed no cytotoxicity. The silicone rubber membrane, which acted as a cell carrier, was compatible with the Endura ElectroForce 3200 mechanical testing instrument, and the mechanical parameters of the loading were controlled via the Bose Peripheral Component Interconnect and WinTest ${ }^{\circledR}$ system (Bose Corp.). The mechanical loading system was able to produce precise deformations on the silicone rubber membrane so that the osteoclasts plated on the membrane were subjected to mechanical stimulation. The reservoir was settled inside a $\mathrm{CO}_{2}$ incubator and the compression pump was operated at a defined speed, so that the medium circulated throughout the culture system via the piping system. This ensured that the mechanical loading system temperature and $\mathrm{CO}_{2}$ concentration were stable (Fig. 1).

Following ethylene oxide sterilization, the silicone rubber membrane was immersed in $\alpha$-Minimum Essential Medium (Shanghai and Shanghai Yu Biotechnology Co., Ltd., Shanghai, China) with 5\% FBS until required. The RAW264.7 cells were seeded at a density of $1 \times 10^{5}$ cells in the silicone rubber membrane with $100 \mathrm{ng} / \mathrm{ml}$ RANKL over the course of five days and were then placed in the mechanical loading system and randomly divided into four groups: A control group (cells were not subjected to any mechanical tension stress) and three experimental groups (cells were subjected to 5, 10 and 15\% stretch microstrain, respectively, at $0.25 \mathrm{~Hz}$ for $1 \mathrm{~h}$ a day). The mechanical loadings continued for three days.

TRAP staining. Three days after the induction of the RAW264.7 cells with $100 \mathrm{ng} / \mathrm{ml}$ RANKL, the osteoclasts were treated with 5,10 and $15 \%$ stretch microstrain for three days (also with $100 \mathrm{ng} / \mathrm{ml}$ RANKL induction), washed with phosphate-buffered saline (PBS) and then fixed with $4 \%$ paraformaldehyde. Following fixation, the cells were rinsed in distilled water and stained using the TRAP staining kit. TRAP-positive cells with two or more nuclei were considered to be osteoclast-like cells. The number of osteoclasts was counted in each film ( $\mathrm{n}=5 /$ group) under a light microscope to observe the effects of mechanical stimulation.

Resorption pit assay. Three days after the induction of the RAW264.7 cells with $100 \mathrm{ng} / \mathrm{ml}$ RANKL, the cells were treated with 5, 10 and $15 \%$ stretch microstrain for three days (also with $100 \mathrm{ng} / \mathrm{ml}$ RANKL induction). The osteoclasts were then seeded on bovine cortical bone slices $(0.5 \times 0.5 \mathrm{~cm})$ at a density of 20,000 cells $/ \mathrm{cm}^{2}$ for three days. The cells were removed from the bovine cortical bone slices by sonication in $0.1 \mathrm{~N} \mathrm{NaOH}$ for $5 \mathrm{~min}$, fixed with $2.5 \%$ glutaraldehyde for $30 \mathrm{~min}$ and then subjected to ethanol gradient dehydration, drying and spraying. The cells were observed using scanning electron microscopy. The surface of each bovine cortical bone slice was examined using light microscopy for 

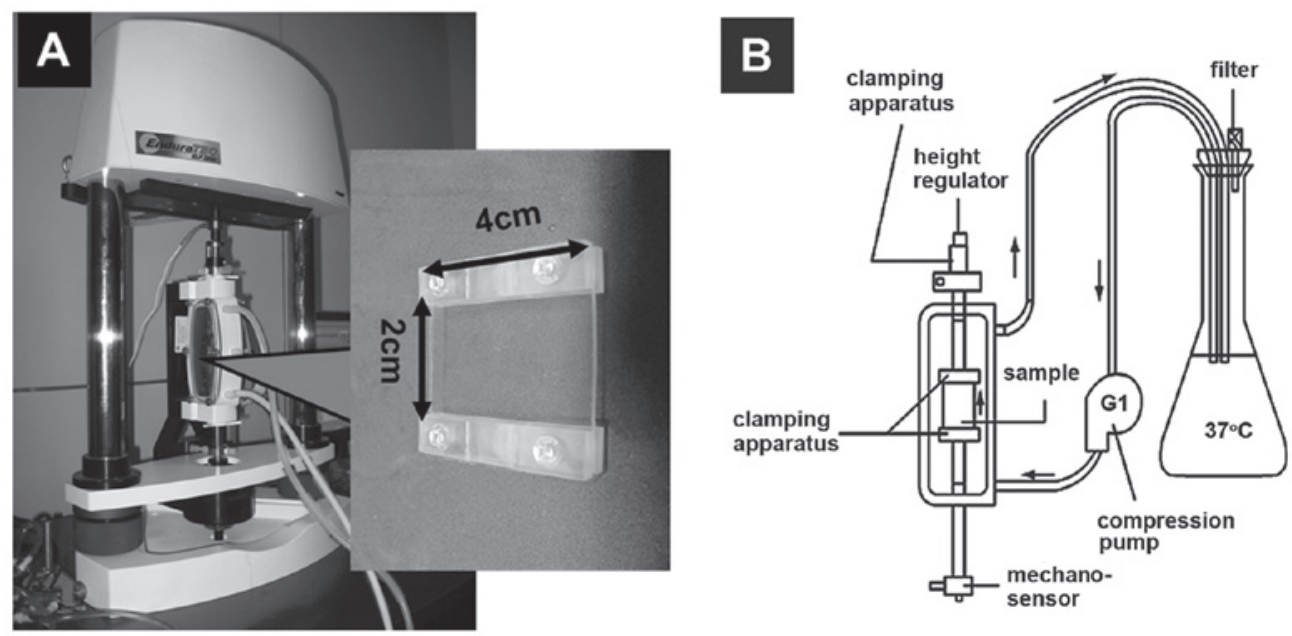

Figure 1. Mechanical loading system. (A) Endura ElectroForce ${ }^{\circledast} 3200$ mechanical testing instrument (Bose Corp., Minnetonka, MN, USA) and the silicone rubber cell carrier. (B) Schematic diagram of the cell strain loading system.

evidence of lacunar resorption, and quantitative analysis of the resorption area was performed with Image-Pro Plus software version 6.0 (Media Cybernetics, Inc., Rockville, MD, USA) ( $n=5 /$ group).

Analysis of osteoclast apoptosis. The effect of mechanical stimulation on osteoclast apoptosis was quantified using the Annexin V-FITC apoptosis detection kit. The osteoclasts were treated with 5, 10 and $15 \%$ stretch microstrain for three days and then washed twice with PBS and gently re-suspended in Annexin V binding buffer at a concentration of $1 \times 10^{6}$ cells $/ \mathrm{ml}$. The osteoclasts $\left(1 \times 10^{5}\right.$ cells, $\left.100 \mu \mathrm{l}\right)$ were added to a $5-\mathrm{ml}$ flow tube, prior to $5 \mu \mathrm{l}$ Annexin V-FITC and $5 \mu \mathrm{l}$ propidium iodide (PI) being transferred. The cells were incubated for $15 \mathrm{~min}$ at room temperature $\left(25^{\circ} \mathrm{C}\right)$ in the dark and then analyzed by flow cytometry within $1 \mathrm{~h}$. Q1 quadrant represented the mechanically damaged cells, Q2 quadrant represented the post-apoptotic or necrotic cells, Q3 quadrant represented the early apoptotic cells and Q4 quadrant represented the surviving cells.

$R T-q P C R$. Following treatment with the mechanical stimuli for three days, the cells were washed twice with PBS and collected. Total RNA was extracted using TRIzol ${ }^{\mathrm{TM}}$ (Invitrogen Life Technologies) according to the manufacturer's instructions. cDNA was synthesized from the total RNA using All-in-One First-Strand cDNA Synthesis kit and oligo(dt) primers (Wuhan Boster Biological Engineering Co., Ltd., Wuhan, China). The primers used for the genetic analysis of the osteoclasts were as follows: Bcl-2 forward, 5'-ACGGGGTGAACTGGG GGAGG-3' and reverse, 5'-GCATGCTGGGGCCGTACA GT-3'; Bax forward, 5'-GATGGACGGGTCCGGAGA-3' and reverse, 5'-CTCAGCCCATCTTCTTCCAG-3'; caspase-3 forward, 5'-TTCAGAGGGGATCGTTGTAGAAGTC-3' and reverse, 5'-CAAGCTTGTCGGCATACTGTTTCAG-3'; cytochrome $c$ forward, 5'-TGGGCGGAAGACAGGTCA-3' and reverse, 5'-TCCAGGGATGTACTTCTTGGGAT-3'; $\beta$-actin forward, 5'-GGGAAATCGTGCGTGACATT-3' and reverse, 5'-GGAACCGCTCATTGCCAAT-3'. The reaction conditions were set according to the kit manufacturer's instructions. Subsequent to the completion of the reaction, amplification and melting curve analyses were performed. Gene expression values were analyzed for target gene expression using the $2^{-\Delta \Delta \mathrm{Ct}}$ method.

Statistical analysis. Data are presented as the mean \pm standard deviation. Statistical analysis was performed with a one-way analysis of variance using SPSS 16.0 software (SPSS, Inc., Chicago, IL, USA). P $<0.05$ was considered to indicate a statistically significant difference.

\section{Results}

Effect of cyclic tension stress on the number of TRAP-positive multinucleated osteoclasts. The effect of cyclic tension stress on osteoclast differentiation was investigated by subjecting RAW264.7 cells to 5, 10 and $15 \%$ stretch microstrain and then counting the number of multinucleated osteoclasts after three days of mechanical stimulation. The number of mature osteoclasts was increased following 10 and $15 \%$ mechanical stimulation $(\mathrm{P}<0.05)$, and the strongest effect was noted following $10 \%$ microstrain (Fig. 2).

Resorption pit assay. As shown in Fig. 3, treatment with mechanical stimulation significantly increased the ability of the experimental group cells to resorb bovine cortical bone slices compared with the control group. No significant difference was observed between the cells subjected to $5 \%$ microstrain and the control cells $(\mathrm{P}>0.05)$.

Cyclic tension stress inhibits osteoclast apoptosis. To determine the effect of cyclic tension stress on osteoclast apoptosis, the Annexin-V/PI binding assay was performed (Fig. 4). Compared with the control group cells $(15.9 \pm 2.36 \%)$, the cells subjected to 5,10 and $15 \%$ stretch microstrain for three days showed significantly decreased early apoptosis (Q3 quadrant) rates (11.47 \pm 1.21 , $8.39 \pm 1.08$ and $10.41 \pm 1.4 \%$, respectively $)(\mathrm{P}<0.05)$. These results indicate that cyclic tension stress inhibits osteoclast apoptosis.

Effect of cyclic tension stress on the expression of osteoclast apoptosis genes. In order to confirm the effect of three days 

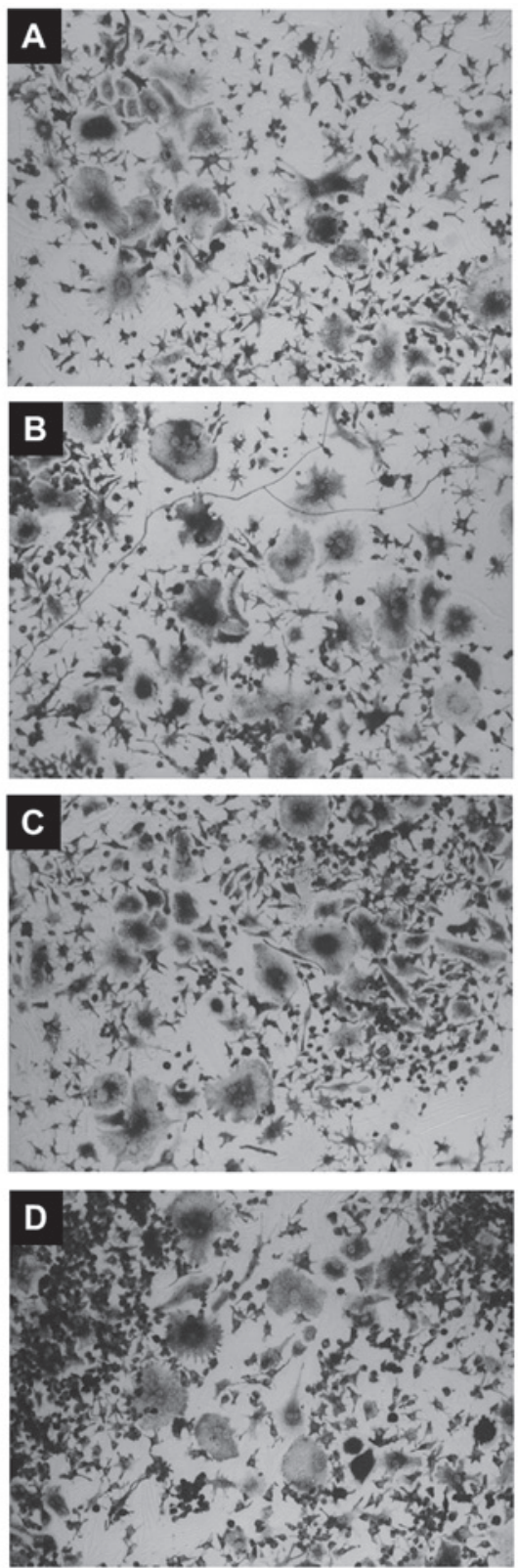

\section{E}

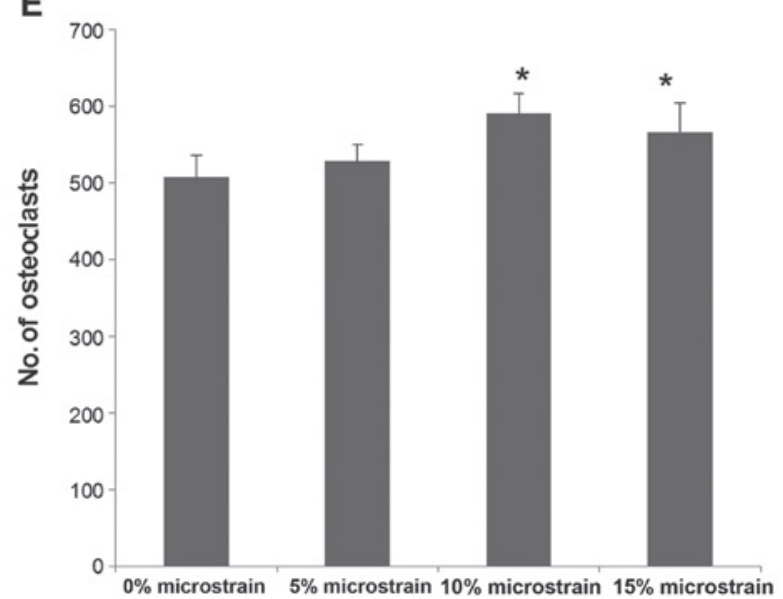

Figure 2. Number of TRAP-positive multinucleated osteoclasts following treatment under different stretch microstrain conditions for three days. (A) Control (0\% stretch microstain); (B) 5\% stretch microstrain (C) $10 \%$ stretch microstrain; (D) 15\% stretch microstrain. (E) Number of TRAP-positive multinucleated cells. Magnification, $\mathrm{x} 100 .{ }^{*} \mathrm{P}<0.05$ vs. the $0 \%$ microstrain group. TRAP, tartrate-resistant acid phosphatase.
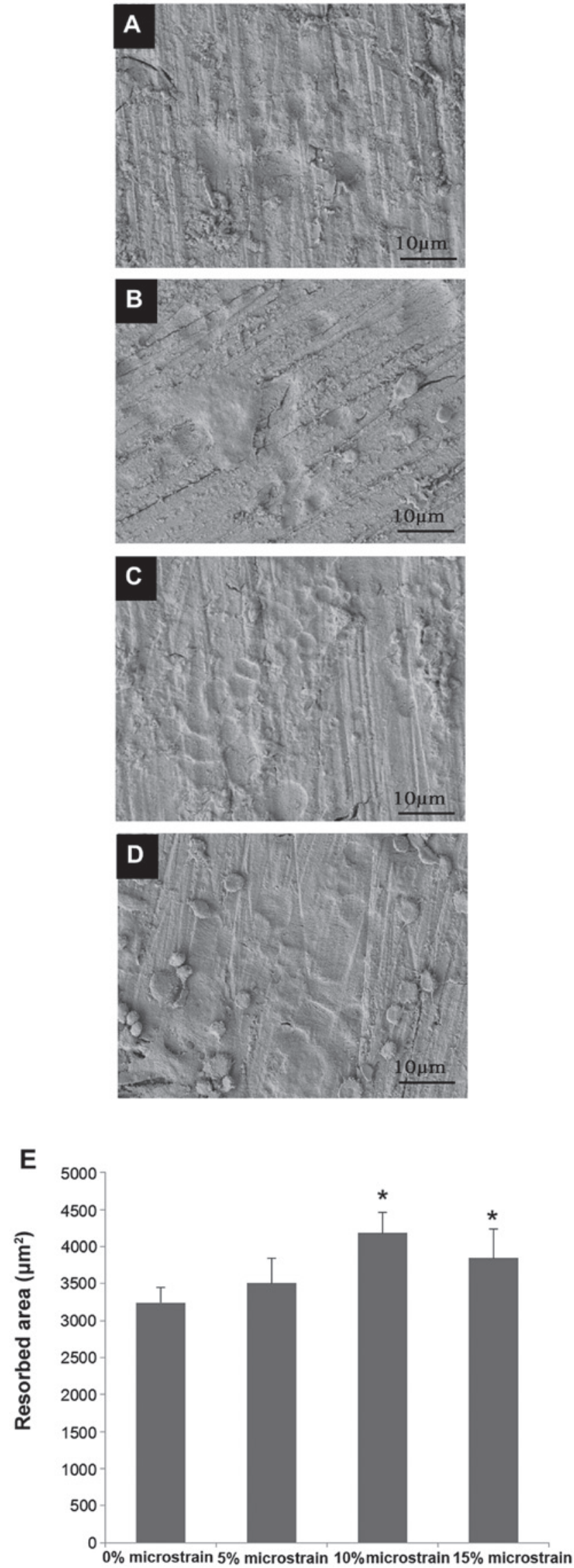

Figure 3. Resorption pit assay results following treatment under different stretch microstrain conditions for three days. (A) Control group (0\% stretch microstain); (B) 5\% stretch microstrain; (C) 10\% stretch microstrain; (D) $15 \%$ stretch microstrain. (E) Analysis of bone resorption area. ${ }^{*} \mathrm{P}<0.05$ vs. the $0 \%$ microstrain group. 


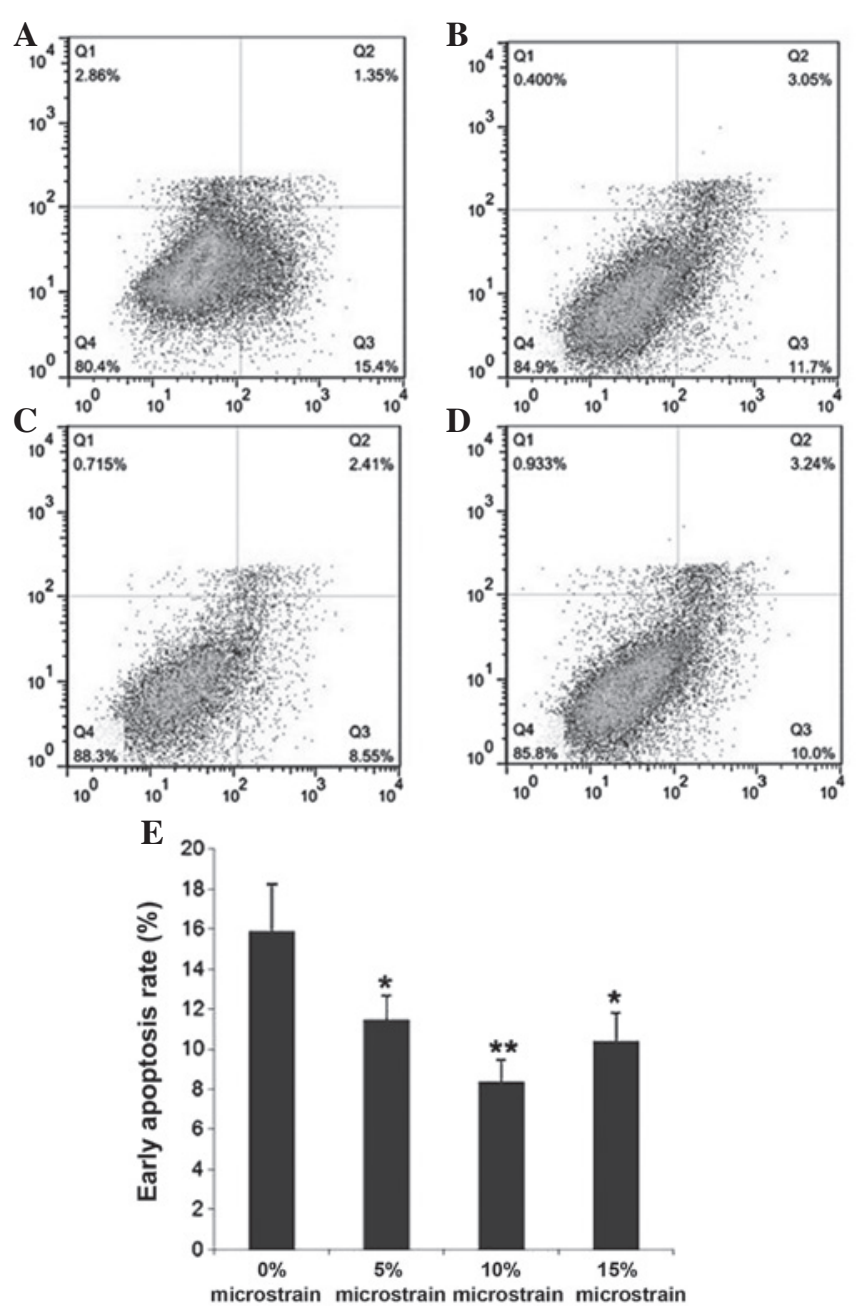

Figure 4. Annexin $\mathrm{V}$ binding assay of osteoclasts following treatment under different stretch microstrain conditions for three days. (A) Control group ( $0 \%$ stretch microstain); (B) 5\% stretch microstrain; (C) 10\% stretch microstrain; (D) $15 \%$ stretch microstrain. (E) Analysis of the early apoptosis rate. Results are presented as the mean \pm standard deviation $(n=5)$. $P<0.05$ and ${ }^{* *} \mathrm{P}<0.01$ vs. the $0 \%$ microstrain group.

of cyclic tension stress on osteoclast apoptosis, RT-qPCR was performed (Fig. 5). Compared with the control group, the mRNA expression of caspase-3 and cytochrome $c$ was decreased in the mechanical loading group $(5,10$ and $15 \%$ stretch microstrain) in a load-dependent manner. The mRNA expression of Bcl-2 showed upregulation and that of Bax showed downregulation. The Bcl-2/Bax ratio of the mechanical stress groups was increased compared with that of the control group, and the increase was greatest in the 10\% stretch microstrain group. These results suggest that cyclic tension stress has an important effect on osteoclast apoptosis.

\section{Discussion}

The present study utilized a novel tension stress loading system designed by our research group. The apoptotic activity of osteoclasts was detected when the cells were subjected to cyclic tension stress during cultivation. The results showed that cyclic tension stress increased the number of TRAP-positive cells and inhibited osteoclast apoptosis. The mRNA expression levels of caspase-3, cytochrome $c$ and Bax were significantly
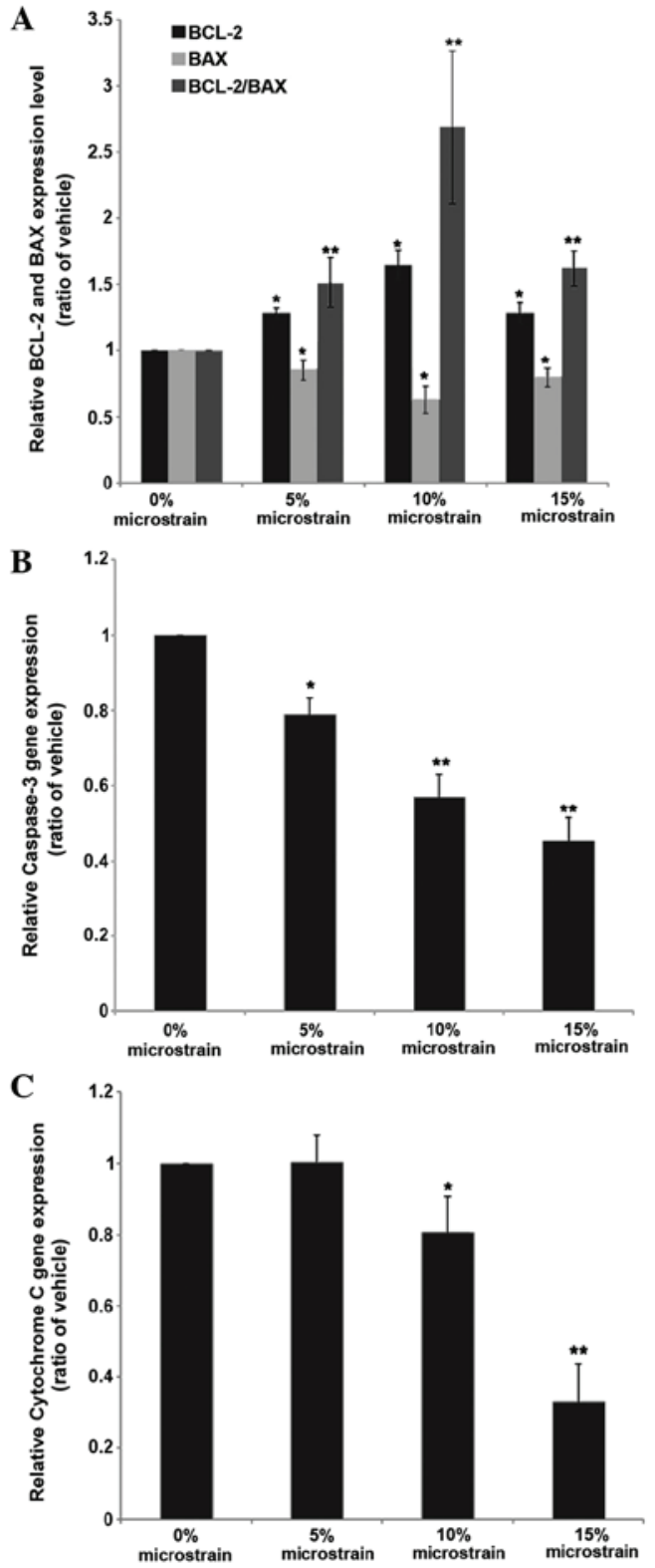

Figure 5. Relative mRNA expression level of osteoclast apoptosis-related genes in the mechanical stress and control groups following treatment under different stretch microstrain conditions for three days. (A-C) mRNA expression of (A) Bcl-2 and Bax, (B) cytochrome $c$ and (C) caspase-3 in osteoclasts. Results are presented as the mean \pm standard deviation $(n=5)$. The control group, which was cultured for the same number of days but without mechanical stress, was defined as the standard $(n=5)$. ${ }^{*} \mathrm{P}<0.05$ and ${ }^{* * *} \mathrm{P}<0.01$ vs. the $0 \%$ microstrain group.

decreased under mechanical stimulation, and the expression of Bcl-2 was increased, suggesting that the apoptotic activity of the osteoclasts was inhibited by the mechanical stimulation.

The advantage of the mechanical loading system used in the present study was that all cells on the silicone rubber membrane were subjected to the same mechanical stimulation, meaning that an effective 'uniaxial' loading was applied to the osteoclasts. In addition, the WinTest software system of the mechanical loading system could control the duration, tensile elongation and tensile frequency of the loading regime, which was important for the cellular responses. The silicone rubber membrane had the three basic characteristics of a cell carrier: 
i) Good transparency, so the microscopic observation of the cells was not affected; ii) good elasticity (the silicone rubber membrane could stretch up to $20 \%$ under the precise control of the operating system); iii) good cytocompatibility.

There were two main methods that could have been used to obtain mature osteoclasts in the present study. Although mature osteoclasts can be isolated from the long bones, the purity is poor and the short life of the cells is not suitable for the needs of the experiment (28-30). Numerous methods have therefore been developed to acquire sufficient osteoclast-like cells in vitro (31-33). In the present study, TRAP-positive, mature osteoclasts with resorptive capabilities were obtained by inducing RAW264.7 cells with $100 \mathrm{ng} / \mathrm{ml}$ RANKL.

Mechanical stimulation plays an important role in the regulation of bone cells. Numerous studies have investigated the effect of mechanical stress on bone tissue in vivo and in vitro $(34,35)$, and it has been reported that the osteoblasts and osteoclasts sense and respond to mechanical stimuli. Studies have confirmed that mechanical stimulation increases the osteoblastic release of ALP (17), NO (18) and PGE2 (19) and regulates Runx2 activation (20). Additionally, studies have reported that mechanical stress can inhibit osteoclast differentiation (23) and increase the activity of bone resorption (24); however, the effect of mechanical stimuli on osteoclast apoptosis as been less well studied. The cell apoptosis pathway is activated by a variety of physical, chemical and biological factors. Apoptosis may occur through activation of the death receptor pathway (Fas/TNF) or the mitochondrial apoptosis pathway (36). The latter is achieved by regulating the expression of the anti-apoptotic protein B-cell lymphoma 2 (Bcl-2) and the pro-apoptotic protein $\mathrm{Bcl} 2$-associated $\mathrm{X}$ protein (Bax) (37). The anti-apoptotic factor Bcl-2 and pro-apoptotic factors (such as Bax) regulated the mitochondrial membrane permeability to decide whether cytochrome $\mathrm{c}$ and other pro-apoptotic factors are release into the cytoplasm.

Apoptosis-related genes of the Bcl-2 family are the key regulators of apoptosis, which exert their effects via the following mechanisms: i) Inhibition of oxygen free radicals; ii) control of intracellular $\mathrm{Ca}^{2+}$ influx, iii) inhibition of the release of cytochrome $c$, iv) inhibition of p53- and c-myc-induced apoptosis. Bax and Bcl-2 are embedded in the mitochondrial membrane. Bax dimers enhance mitochondrial membrane permeability, while Bcl-2 and Bax can combine to form a heterodimeric body, which prevents the formation of pro-apoptotic Bax dimers (37). Bcl-2 dimers can additionally inhibit mitochondrial depolarization (38-40). When cytochrome $c$ is released into the cytoplasm, it can combine with apoptosis protease activating factor-1 (APAF-1) and enhance the combined capacity of APAF-1 and adenosine triphosphate. Recruitment of the caspase- 9 precursor molecule to the APAF-1 complex results in caspase- 9 activation. The effectors caspase-3, -6 and -7 are then gradually activated, furthering the cells along the apoptotic pathway (41-43). The caspase family of cysteine aspartic proteases plays an important role at the start and finish of cell apoptosis and acts as the executors of cell apoptosis. Two pathways can result in caspase activation. In the first pathway, caspase activation is mediated by death receptors, such as Fas or the TNF receptor. Fas ligand and Fas receptor combine, and then Fas-Associated protein with Death Domain binds to the receptor, leading to pro-caspase- 8 combination and automatic activation. Caspase-3 and other downstream caspases are then activated, which in turn lead to a caspase cascade amplification reaction, cracking protein substrates in the cell (44). The second pathway leading to caspase activation begins with pro-apoptotic signals promoting cytochrome $c$ release from the mitochondria into the cytosol, where it enters the cytoplasm, combines with APAF-1 and then binds to and activates pro-caspase- 9 . The composite activated caspase-3 leads to the caspase cascade amplification. Caspase- 3 acts as the converging point of a variety of apoptosis-stimulating signals and the ultimate enforcer of cell apoptosis. Activation of caspase- 3 is representative of the cell reaching the irreversible phase of apoptosis (45).

In the present study, it was demonstrated that cyclic tension stress could upregulate the mRNA expression of Bcl-2 and downregulate that of Bax, thus increasing the $\mathrm{Bcl}-2 / \mathrm{Bax}$ ratio. This reduced the permeability of the mitochondrial membrane and suppressed the release of cytochrome $c$ and other pro-apoptotic factors into the cytoplasm. The final caspase cascade mediating the apoptosis of osteoclasts was inhibited. Changes in the deformation variables affected each gene differently; for example, $10 \%$ stretch microstrain elicited the strongest effect on the expression of Bcl-2 and Bax and the Bcl-2/Bax ratio, while the expression levels of caspase-3 and cytochrome $c$ were inhibited most strongly by $15 \%$ stretch microstrain. The results suggest that mild mechanical stimulation $5 \%$ stretch microstrain) has only a slight effect on osteoclast apoptosis and that moderate mechanical stimulation (10\% stretch microstrain) inhibits osteoclast apoptosis mainly by regulating the ratio of Bcl-2/Bax but also by inhibiting the expression of cytochrome $c$ and caspase-3. By contrast, high levels of mechanical stimulation (15\% stretch microstrain) regulate osteoclast apoptosis mainly by inhibiting the expression of cytochrome $c$ and caspase-3, and secondly by regulating the ratio of Bcl-2/Bax.

In conclusion, cyclic tension stress can inhibit osteoclast apoptosis through the mitochondria-mediated apoptosis pathway. The mechanism by which stretch microstrain stimuli affect osteoclast apoptosis varies depending on the size of the stimulus. Since osteoblasts and osteoclasts are closely associated in vivo, the experimental study of the mechanical regulation of osteocytes requires further development.

\section{Acknowledgements}

This study was funded by the Public Health Bureau of Science and Technology of Tianjin, China (no. 2011KZ57) and the Traditional Chinese Medicine Administration of Tianjin, China (no. 13123).

\section{References}

1. Nakahama K: Cellular communications in bone homeostasis and repair. Cell Mol Life Sci 67: 4001-4009, 2010.

2. Liu $\mathrm{H}$ and Li B: p53 control of bone remodeling. J Cell Biochem 111: 529-534, 2010.

3. Ehrlich PJ and Lanyon LE: Mechanical strain and bone cell function: A review. Osteoporos Int 13: 688-700, 2002.

4. Chow JW, Jagger CJ and Chambers TJ: Reduction in dynamic indices of cancellous bone formation in rat tail vertebrae after caudal neurectomy. Calcif Tissue Int 59: 117-120, 1996.

5. Huiskes R, Ruimerman R, van Lenthe GH and Janssen JD: Effects of mechanical forces on maintenance and adaptation of form in trabecular bone. Nature 405: 704-706, 2000. 
6. Rubin CT and Lanyon LE: Kappa Delta Award paper Osteoregulatory nature of mechanical stimuli: Function as a determinant for adaptive remodeling in bone. J Orthop Res 5: 300-310, 1987.

7. Jee WS, Li XJ and Schaffler MB: Adaptation of diaphysea structure with aging and increased mechanical usage in the adult rat: A histomorphometrical and biomechanical study. Anat Rec 230: 332-338, 1991

8. Turner CH, Akhter MP, Raab DM, Kimmel DB and Recker RR A noninvasive, in vivo model for studying strain adaptive bone modeling. Bone 12: 73-79, 1991

9. Chambers TJ, Evans M, Gardner TN, Turner-Smith A and Chow JW: Induction of bone formation in rat tail vertebrae by mechanical loading. Bone Miner 20: 167-178, 1993.

10. Forwood MR and Turner CH: Skeletal adaptations to mechanical usage: Results from tibial loading studies in rats. Bone 17 (4 Suppl): 197S-205S. 1995

11. Mosley JR, March BM,Lynch J and Lanyon LE: Strain magnitude related changes in whole bone architecture in growing rats Bone 20: 191-198, 1997.

12. Cowin SC, Moss-Salentijn L and Moss ML: Candidates for the mechanosensory system in bone. J Biomech Eng 113: 191-197, 1991

13. Weinbaum S, Cowin SC and Zeng Y: A model for the excitation of osteocytes by mechanical loading-induced bone fluid shear stresses. J Biomech 27: 339-360, 1994.

14. Kaspar D, Seidl W, Neidlinger-Wilke C, et al: Proliferation of human-derived osteoblast-like cells depends on the cycle number and frequency of uniaxial strain. J Biomech 35: 873-880, 2002.

15. Kaspar D, Seidl W, Neidlinger-Wilke C, et al: Dynamic cell stretching increases human osteoblast proliferation and CICP synthesis but decreases osteocalcin synthesis and alkaline phosphatase activity. J Biomech 33: 45-51, 2000.

16. Neidlinger-Wilke C, Wilke HJ and Claes L: Cyclic stretching of human osteoblasts affects proliferation and metabolism: A new experimental method and its application. J Orthop Res 12: 70-78, 1994.

17. Chen YJ, Huang CH, Lee IC, et al: Effects of cyclic mechanical stretching on the mRNA expression of tendon/ligament-related and osteoblast-specific genes in human mesenchymal stem cells Connect Tissue Res 49: 7-14, 2008.

18. Hara F, Fukuda K, Ueno M, et al: Pertussis toxin-sensitive $\mathrm{G}$ proteins as mediators of stretch-induced decrease in nitric-oxide release of osteoblast-like cells. J Orthop Res 17: 593-597, 1999.

19. Searby ND, Steele CR and Globus RK: Influence of increased mechanical loading by hypergravity on the microtubule cytoskeleton and prostaglandin E2 release in primary osteoblasts. Am J Physiol Cell Physiol 289: C148-C158, 2005.

20. KannoT,TakahashiT,TsujisawaT, et al:Mechanical stress-mediated Runx2 activation is dependent on Ras/ERK1/2 MAPK signaling in osteoblasts. J Cell Biochem 101: 1266-1277, 2007.

21. Boyle WJ, Simonet WS and Lacey DL: Osteoclast differentiation and activation. Nature 423: 337-342, 2003.

22. Quinn JM and Gillespie MT: Modulation of osteoclast formation. Biochem Biophys Res Commun 328: 739-745, 2005.

23. Suzuki N, Yoshimura Y, Deyama Y, et al: Mechanical stress directly suppresses osteoclast differentiation in RAW264.7 cells. Int J Mol Med 21: 291-296, 2008.

24. Kurata K, Uemura T, Nemoto A, et al: Mechanical strain effect on bone-resorbing activity and messenger RNA expressions of marker enzymes in isolated osteoclast culture. J Bone Mine Res 16: 722-730, 2001.

25. Weinstein RS and Manolagas SC: Apoptosis and osteoporosis. Am J Med 108: 153-164, 2000
26. Abe K, Yoshimura Y, Deyama Y, et al: Effects of bisphosphonates on osteoclastogenesis in RAW264.7 cells. Int J Mol Med 29: 1007-1015, 2012.

27. Arriero Mdel M, Ramis JM, Perelló J and Monjo M: Inositol hexakisphosphate inhibits osteoclastogenesis on RAW 264.7 cells and human primary osteoclasts. PLoS One 7: e43187, 2012.

28. Osdoby P, Martini MC and Caplan AI: Isolated osteoclasts and their presumed progenitor cells, the monocyte, in culture. J Exp Zool 224: 331-344, 1982.

29. Zambonin Zallone A, Teti A and Primavera MV: Isolated osteoclasts in primary culture: First observations on structure and survival in culture media. Anat Embryol (Berl) 165: 405-413, 1982.

30. Tezuka K, Tezuka Y, Maejima A, et al: Molecular cloning of a possible cysteine proteinase predominantly expressed in osteoclasts. J Biol Chem 269: 1106-1109, 1994.

31. Udagawa $\mathrm{N}$, Takahashi $\mathrm{N}$, Akatsu $\mathrm{T}$, et al: The bone marrow-derived stromal cell lines MC3T3-G2/PA6 and ST2 support osteoclast-like cell differentiation in cocultures with mouse spleen cells. Endocrinology 125: 1805-1813, 1989.

32. Collin-Osdoby P, Oursler MJ, Webber D and Osdoby P: Osteoclast-specific monoclonal antibodies coupled to magnetic beads provide a rapid and efficient method of purifying avian osteoclasts. J Bone Miner Res 6: 1353-1365, 1991.

33. Quinn JM, Neale S, Fujikawa Y, et al: Human osteoclast formation from blood monocytes, peritoneal macrophages, and bone marrow cells. Calcif Tissue Int 62: 527-531, 1998.

34. Duncan RL and Turner $\mathrm{CH}$ : Mechanotransduction and the functional response of bone to mechanical strain. Calcif Tissue Int 57: 344-358, 1995.

35. Skerry TM and Suva LJ: Investigation of the regulation of bone mass by mechanical loading: From quantitative cytochemistry to gene array. Cell Biochem Funct 21: 223-229, 2003.

36. Kroemer G, Galluzzi L and Brenner C: Mitochondrial membrane permeabilization in cell death. Physiol Rev 87: 99-163, 2007

37. Adams JM and Cory S: Bcl-2-regulated apoptosis: Mechanism and therapeutic potential. Curr Opin Immunol 19: 488-496, 2007.

38. Yin XM: Bid, a BH3-only multi-functional molecule, is at the cross road of life and death. Gene 369: 7-19, 2006.

39. Certo M, Del Gaizo Moore V, Nishino M, et al: Mitochondria primed by death signals determine cellular addiction to antiapoptotic BCL-2 family members. Cancer Cell 9: 351-365, 2006.

40. Kuwana T and Newmeyer DD: Bcl-2-family proteins and the role of mitochondria in apoptosis. Curr Opin Cell Biol 15: 691-699, 2003.

41. Hellebrand EE and Varbiro G: Development of mitochondrial permeability transition inhibitory agents: A novel drug target. Drug Discov Ther 4: 54-61, 2010.

42. Jilka RL, Noble B and Weinstein RS: Osteocyte apoptosis. Bone 54: 264-271, 2013.

43. Timmer JC and Salvesen GS: Caspase substrates. Cell Death Differ 14: 66-72, 2007.

44. Dai C, Zhang B, Liu X, et al: Pyrimethamine sensitizes pituitary adenomas cells to temozolomide through cathepsin B-dependent and caspase-dependent apoptotic pathways. Int J Cancer 133: 1982-1993, 2013

45. Cryns V and Yuan J: Proteases to die for. Genes Dev 12: 1551-1570, 1998. 